\title{
High-Speed, Solid State, Interferometric Interrogator and Multiplexer for Fibre Bragg Grating Sensors
}

\author{
Philip Orr, Student Member, IEEE, Paweł Niewczas, Member, IEEE
}

\begin{abstract}
We report on the design and prototyping of a robust high-speed interferometric multiplexer and interrogator for fibre Bragg grating sensors. The scheme is based on the combination of active WDM channel switching and passive, instantaneous interferometry, allowing the resolution of virgin interferometric interrogators to be retained at $\mathrm{MHz}$ multiplexing rates. In this article the system design and operation are described, and a prototype scheme is characterised for three sensors and a multiplexing rate of $4 \mathrm{kHz}$, demonstrating a noise floor of 10 $\mathrm{n \varepsilon} / \sqrt{ } \mathrm{Hz}$ and no cross-sensitivity. It is proposed that the system will be applicable to demanding monitoring applications requiring high speed and high resolution measurements across the sensor array.
\end{abstract}

Index Terms-fibre Bragg grating, interrogation, sensors, interferometry, structural monitoring.

\section{INTRODUCTION}

$\mathbf{F}$ IBRE Bragg gratings (FBGs) are periodic perturbations of the refractive index along a fibre core, having peak optical reflection at a specific wavelength known as the Bragg wavelength [1]. In sensor applications, their wavelengthencoding nature coupled with their simple reflected spectra mean that FBGs are relatively easy to interrogate and multiplex, and are effectively immune to the problems of intensity fluctuations and attenuation [2]. For these reasons the FBG is now ubiquitous in the field of optical instrumentation, and may be credited with making a large contribution to the increasing market presence of optical fibre sensors [3,4].

Interrogation schemes for FBG sensors require precise detection and location of the FBG centre wavelength, which shifts with changes in various external parameters - most commonly axial strain and temperature [2]. Since serial multiplexing of FBG sensors along a single fibre may be achieved by writing FBGs at unique Bragg wavelengths, the multiplexing technique is a key component of FBG interrogation systems. While FBG wavelength detection has been realised by a number of methods (including interferometry and prism/CCD arrays [5]), the most common uses a combination of a broadband light source, tunable filter

Manuscript received Aug $19^{\text {th }}$, 2011. This work was supported by the Engineering and Physical Sciences Research Council.

P. Orr and P. Niewczas are with the Institute for Energy and Environment, University of Strathclyde, 204 George Street, Glasgow, G1 1XW, UK. (phone: +44 (0)141 548 4841; e-mail: philip.orr@strath.ac.uk) (either scanning Fabry-Perót or acousto-optic [6]) and photodetector, such that wavelength division multiplexing of serially arranged gratings can be achieved straightforwardly [7]. A scanning laser may alternatively be employed to provide identical functionality. Such "tuned filter" schemes require precise control of the tunable spectral component to achieve satisfactory peak detection resolution and accuracy, and high levels of performance are difficult to achieve in practice due to inherent nonlinearities, drifts, and electronic noise - particularly at higher scanning frequencies.

An alternative, highly precise peak detection technique employing interferometry is often used, as described by Kersey et al [8]. Interferometric interrogation, while offering the highest resolution of all techniques, is difficult to integrate with existing wavelength multiplexing schemes due to the requirement for either stabilisation or modulation of the optical path difference (OPD). As such, it is most commonly utilised in single-sensor schemes for high-frequency, lowamplitude measurements. Conventional interrogation systems therefore possess a trade-off between their extensibility (number of sensors the scheme can interrogate) and the quality of the measurement (accuracy, resolution, and rate of measurement).

Hybrid interferometric/WDM schemes have, however, been successfully developed in attempts to address this trade-off. Of particular note are the schemes by Berkoff [9] and Johnson [10]; the former combining passive WDM with active OPD modulation, and the latter utilising an instantaneous phase demodulation technique [11] in order to deploy a continuously-scanning spectral filter. These schemes however retain performance limitations stemming from the need for active OPD modulation, or the use of tunable components and substantial electronic processing stages, resulting in increased noise levels and speed reductions compared with virgin interferometric techniques. In particular, the use of a tunable filter limits the achievable interrogation rate and is uneconomical with time during the scanning operation.

In this article we describe an interferometric multiplexing interrogator for FBG sensors which incorporates minimal electronics, retains the resolution of simple interferometric schemes, and enables unprecedented measurement rates. Based on a fast optical path switch and passive WDM module, the scheme is simple and robust with no tuned or modulated components, and is able to fully exploit the immediacy of the passive phase demodulation technique. The proposed scheme 


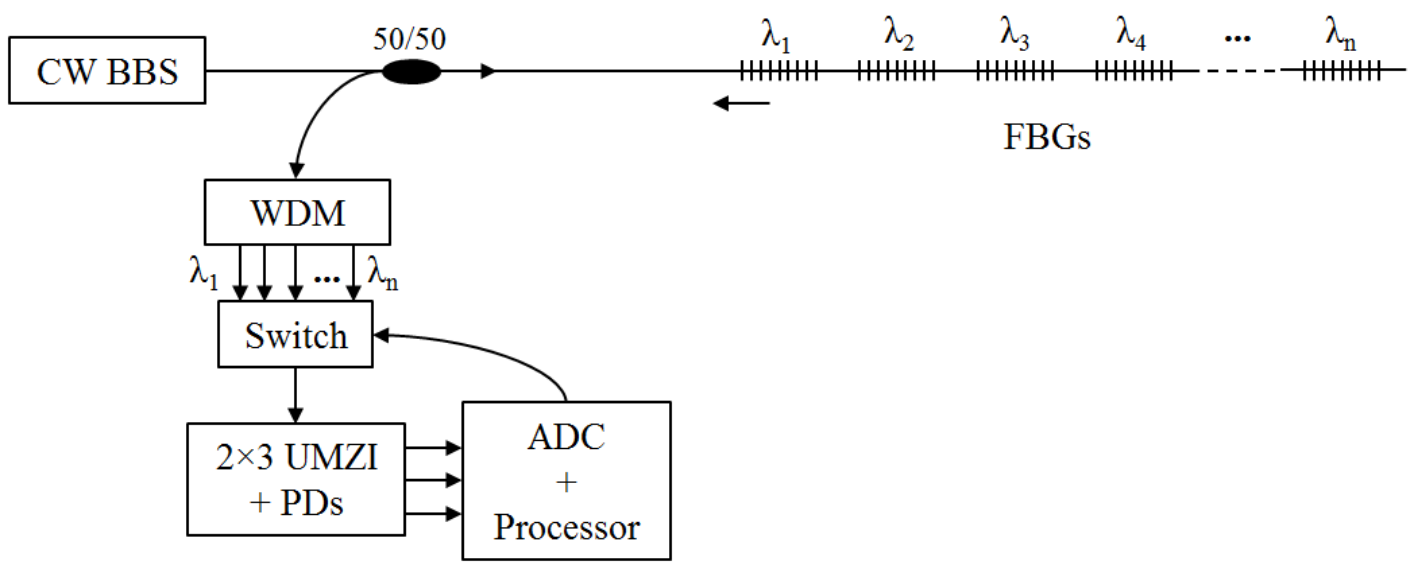

Fig. 1. General architecture of the fast solid-state interrogation system for measurement of quasistatic or dynamic shifts in FBG sensor wavelengths. $B B S$ is broadband source, WDM is passive wavelength division multiplexer. The block $2 \times 3 U M Z I+P D s$ is detailed in Fig. 2. ADC + Processor functionality is described in Fig. 3.

will thus be applicable to high-performance applications for which both high-speed and high-resolution measurements are required over the entire sensor array.

\section{SYSTEM DESIGN}

Fig. 1 illustrates the proposed interrogation scheme. A CW broadband source is used to illuminate an array of seriallymultiplexed FBG sensors, each of which reflects at a unique wavelength $\lambda_{1}-\lambda_{\mathrm{n}}$ within the source bandwidth. Upon returning, sensor reflections are coupled into a series of modules, described hereafter, for active sensor selection and passive wavelength shift demodulation.

\section{A. Instantaneous phase demodulation}

The proposed scheme exploits a passive, instantaneous technique for phase demodulation based on the physical characteristics of single-fusion $3 \times 3$ couplers. As described by Todd in [12], by fabricating a Mach-Zehnder interferometer (MZI) with a known path difference between a $2 \times 2$ and a $3 \times 3$ coupler, the phase difference may be calculated ratiometrically based on the photodetected outputs of all three channels. Fig. 2 illustrates the unbalanced MZI module with integrated photodetectors that is used in this scheme.

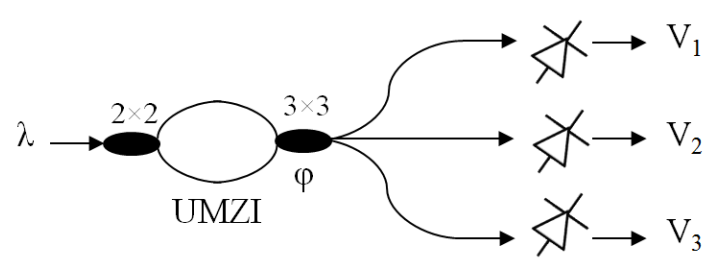

Fig. 2. Core demodulation platform: An input optical wavelength is converted to a phase difference within the unbalanced two-path interferometer (UMZI). The outputs of the single-fusion $3 \times 3$ coupler are used to determine the phase difference by the ratio of their optical powers (voltages $V_{1-3}$ after photodetection).

The phase difference $\phi$ between MZI paths is related to the input optical wavelength $\lambda$ (in this scheme the reflected Bragg wavelength) by the expression

$$
\phi=\frac{2 \pi n d}{\lambda}
$$

where $n$ is the MZI core index and $d$ is the physical path difference.

By making the $3 \times 3$ coupler the point of interference of the two paths, the optical power at the three MZI outputs represent the same interference pattern spaced mutually by $120^{\circ}$. After photodetection the corresponding voltage outputs can be described most concisely as

$$
V_{n}=a_{n}+b_{n} \cos \left(\phi+\theta_{n}\right)
$$

where $a_{n}$ and $b_{n}$ are the amplitudes of the dc and ac components of the interference fringes respectively, and $\theta_{n}$ is the initial phase (nominally $0^{\circ}, 120^{\circ},-120^{\circ}$ for $\mathrm{n}=1,2,3$ ). By modulating the interferometer by at least $2 \pi$ during initial system calibration, the above values can be determined for each of the three output channels using an established technique [13]. If normalised constants are prepared $\left(\alpha_{n}=a_{n} / a_{1}\right.$ and $\left.\beta_{n}=b_{n} / b_{1}\right)$ the phase can then be calculated using the equation

$$
\phi(t)=\tan ^{-1}\left[\frac{\left(\mu_{2}-\mu_{3}\right) V_{1} / \alpha_{1}+\left(\mu_{3}-\mu_{1}\right) V_{2} / \alpha_{2}+\left(\mu_{1}-\mu_{2}\right) V_{3} / \alpha_{3}}{\left(\gamma_{2}-\gamma_{3}\right) V_{1} / \alpha_{1}+\left(\gamma_{3}-\gamma_{1}\right) V_{2} / \alpha_{2}+\left(\gamma_{1}-\gamma_{2}\right) V_{3} / \alpha_{3}}\right]
$$

where $\gamma_{n}=\beta_{n} \sin \left(\theta_{n} / \alpha_{n}\right)$ and $\mu_{n}=\beta_{n} \cos \left(\theta_{n} / \alpha_{n}\right)$ are normalising parameters containing mean intensity level, fringe visibility, photodetector responsivity, and the properties of both couplers used to form the interferometer, and $V_{1}, V_{2}$ and $V_{3}$ are the outputs of photodetectors from Fig. 2. It is important to note that by using the normalised constants $\alpha_{\mathrm{n}}$ and $\beta_{\mathrm{n}}$, the calculation of the phase $\phi$ is immune to commonmode disturbances such as fluctuations in optical power.

Using this technique, an optical wavelength guided to the input of the MZI can be determined immediately from a single 
sample on each $\mathrm{V}_{1-3}$. This capability for immediate calculation of the reflected wavelength from an FBG sensor is key to the operation of the full scheme described here.

\section{B. Switched-band FBG multiplexing}

In the proposed system, the FBG wavelengths and CWDM channel centre wavelengths are matched such that each channel of the WDM module is associated with a particular FBG. Therefore, every FBG wavelength must be unique and must correspond to a single WDM channel. This allows the scheme to exploit the immediacy of the phase demodulation technique by selecting, at rates limited only by switching speeds and processing capability, which sensor reflection is guided to the interferometer for detection. Commercially available switches are presently capable of switching between 16 channels at rates of up to $10 \mathrm{MHz}$ (with an attenuation/settling time of around $2.5 \mathrm{~ns}$ ), e.g. [14]. Typically, such devices incorporate a $20 \mathrm{~dB}$ extinction ratio between channels that is consistent up to the maximum switching rate, beyond which cross-talk increases and the device becomes unfit for purpose. Combined with established arrayed-waveguide grating technology, wavelength switches as required for the proposed scheme are readily constructed using off-the-shelf components.

As stated, the continuous wave broadband source illuminates a wavelength range covering the reflection wavelengths of all sensors within the sensor array. In the present embodiment, reflected wavelengths from all sensors return simultaneously and continuously to the WDM module, which passively separates off signals from each sensor onto independent output fibres. An optical path switch, driven by the ADC/Processor unit, is used to guide the reflected signal of each sensor in turn to the demodulation platform where the voltages $V_{1-n}$ are immediately sampled by the ADC unit and thereafter processed to determine the FBG wavelength. The wavelength switch can select between sensor signals in any order. In addition, the ADC sampling rate may be set to any integer multiple of the switching rate to enable oversampling for the purposes of electronic noise processing and hence further improved measurement resolution. In this way, additional processing in the form of peak hold circuitry, used in previous hybrid interrogators [10] is not required, improving the potential measurement resolution and simplicity of system construction.

\section{Operational procedure and processing}

Fig. 3 depicts the processing and control cycle of the interrogation scheme. For each path switch state (which may be driven between sensors in any sequence) the analogue voltages $V_{1-3}$ are sampled and digitized, and thereafter the phase change $\Delta \phi$ is calculated using (3). For each phase calculation, an appropriate arctangent unwrapped scheme should be used, such as that described in [12]. A Boolean flag may be set at this stage to indicate the present state of wrapping. In the next occurrence of this sensor, the present and previous wrap states may be compared to determine if wrapping has occurred and the appropriate magnitude of unwrapping that should be applied. This procedure is described in more detail in [12].
Thus, the phase value for each sensor is calculated and stored between each path switch operation. Alternatively, the photodetector voltages may be stored for processing offline. As an example of a common measurement application, following the calculation of $\Delta \phi$ the longitudinal strain in the grating may be calculated using the expression

$$
\Delta \varepsilon=\frac{\lambda}{2 \pi n d \zeta} \Delta \phi
$$

which is obtained by differentiating (1) with respect to $\lambda$ and substituting in $\zeta=(\delta \lambda / \delta \varepsilon) / \lambda\left(0.74 \times 10^{-6} \mu \varepsilon^{-1}\right.$ [15]), which relates longitudinal strain in the fibre to Bragg wavelength shift.

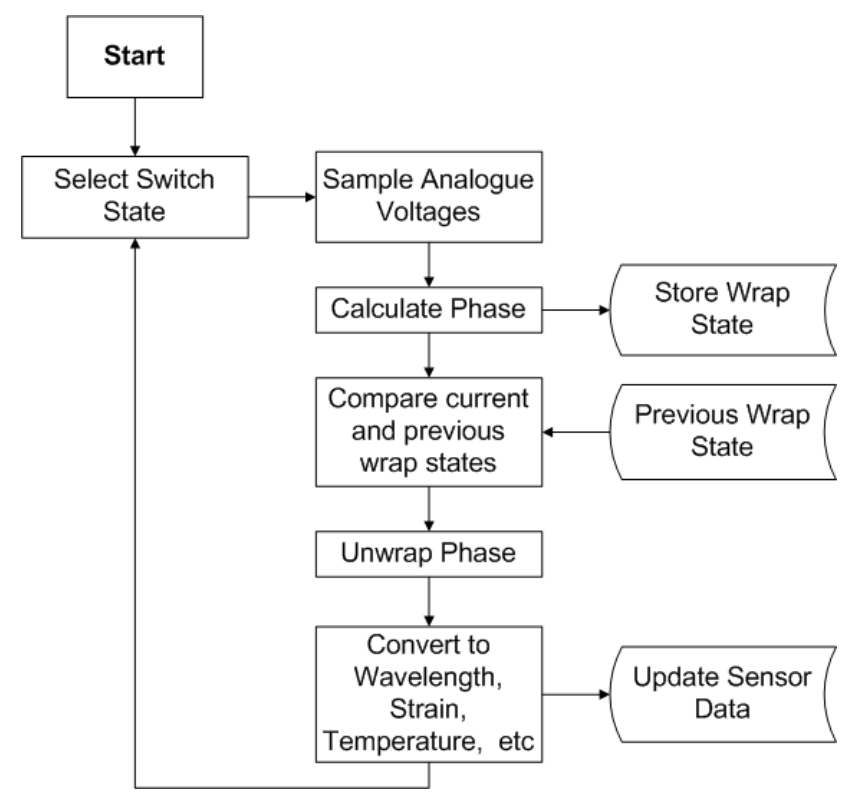

Fig. 3. Flowchart of $A D C /$ Processor block operation incorporating phase unwrapping and data storage. Software control of the wavelength switch allows sensors to be interrogated in any sequence and with variable sampling rates.

The phase-wavelength sensitivity is governed by the OPD in the interferometer (nd in (4)). For the majority of sensing applications it will be beneficial to maximise the OPD in order to increase sensitivity. However, it has been demonstrated that in the preferred passive phase demodulation technique the OPD and hence sensitivity to Bragg shifts has a practical maximum dictated not only by FBG coherence length but also by demodulation errors introduced by electronic noise as the fringe spacing reduces below the FBG bandwidth. This error has been shown to increase proportionally with the OPD value [16]. Therefore, in the laboratory prototype we limit the OPD to approximately $3 \mathrm{~mm}$.

\section{CONSTRUCTION AND CHARACTERISATION}

\section{A. Prototype construction and test arrangement}

In order to demonstrate the capability of the scheme, a small-scale prototype was constructed using a 4-channel WDM unit and a $1 \times 4$ bidirectional optical path switch with a 
maximum switching repetition rate of $5 \mathrm{KHz}$ (Agiltron Inc.). The WDM channel centres were spaced at $10 \mathrm{~nm}$ intervals $(1535,1545,1555,1565 \mathrm{~nm})$.

A serially-multiplexed array of three FBG sensors with nominal Bragg wavelengths of 1535, 1545 and $1555 \mathrm{~nm}$ was used to test the interrogator. As illustrated in Fig. 4, each FBG sensor was bonded to piezoelectric stacks such that longitudinal strain may be translated to the sensing fibre by applying a voltage difference over each stack. These three piezoelectric stacks were then actuated by 3-phase mains voltage via a variable transformer. The induced $50 \mathrm{~Hz}$ ac strains were therefore mutually phase-separated by $120^{\circ}$.

The unbalanced MZI (UMZI in Fig. 4), incorporating an optical path difference ( $n d$ ) of $3 \mathrm{~mm}$, is an all-fibre construction fabricated between the output pigtails of $1 \times 2$ and $3 \times 3$ couplers by fusion splicing. The overall lengths of the interferometer arms were approximately $10 \mathrm{~cm}$ to allow for splicing. To isolate the MZI from vibrations, the device was placed on a metal plate with two grooves machined to accommodate coupler packaging. MZI arms were then bonded to the plate with a suitable epoxy.

In order to acquire measurements from all FBGs in a continuous sequence, the internal clock of the ADC unit (National Instruments PXI) was used to clock a 2-bit counter IC (74LS161) and also to trigger switching and sampling as shown in Fig. 4. This counter provides the setting bits for the 4-way path switch, clocking on a TTL rising edge. On the falling edge, the switch is reconfigured to the new settings.
The rising edge is also used to trigger digitization of the three analogue photodetector voltages. In this way, a single clock is used to cycle gratings and acquire voltages in a staggered fashion. Processing of voltages $V_{1-3}$ using (3) and (4) and subsequent data sorting, storage and display is then performed in software on a desktop computer.

The total size of the prototype interrogator - including source, WDM module, path switch, interferometer, and electronics - permits housing in standard 19" rack mount enclosures. The system incorporates no moving parts, requires minimal heat dissipation, and has no high voltage isolation requirements.

\section{B. Measurement and multiplexing performance}

During testing, the ADC unit was configured for $4 \mathrm{kHz}$ clock rate, yielding path switching at $4 \mathrm{kHz}$ and hence $1 \mathrm{kHz}$ acquisition rate per sensor. The $4^{\text {th }}$ channel, with no corresponding FBG, was ignored during these tests. Figs. 5 (a)-(c) show signals from each sensor during the application of 3-phase $50 \mathrm{~Hz}$ ac voltage to the piezoelectric stacks, resulting in rms strains of 50-70 $\mu \varepsilon$ depending on the piezoelectric stack characteristics. The $120^{\circ}$ shift between applied voltage phasors can be clearly seen between demodulated phase signals.

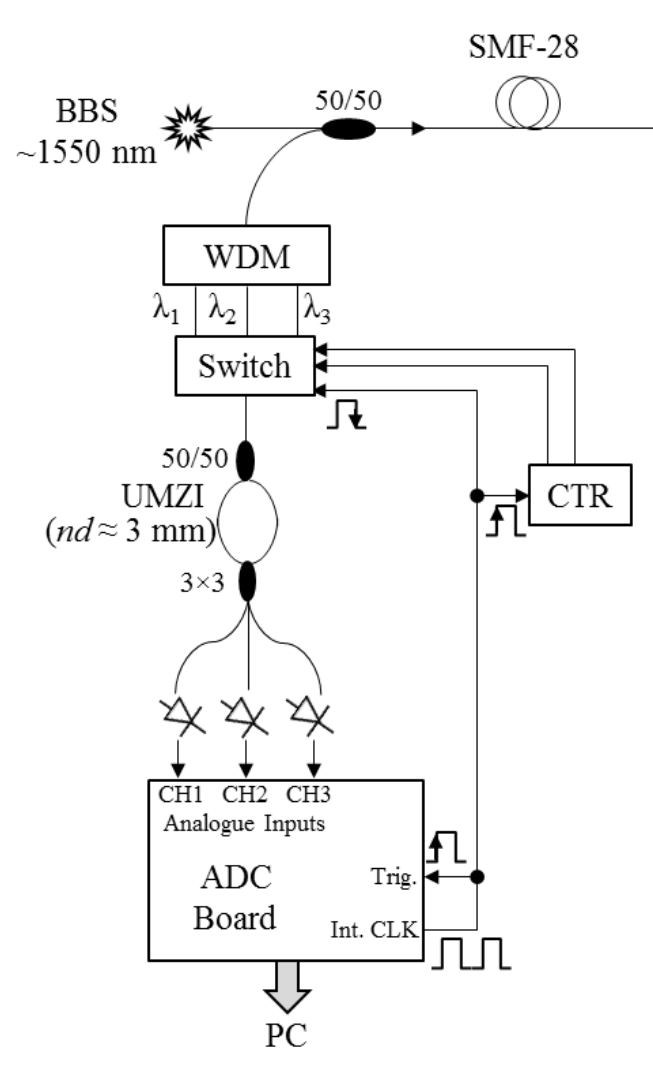

(a)
FBG Sensors

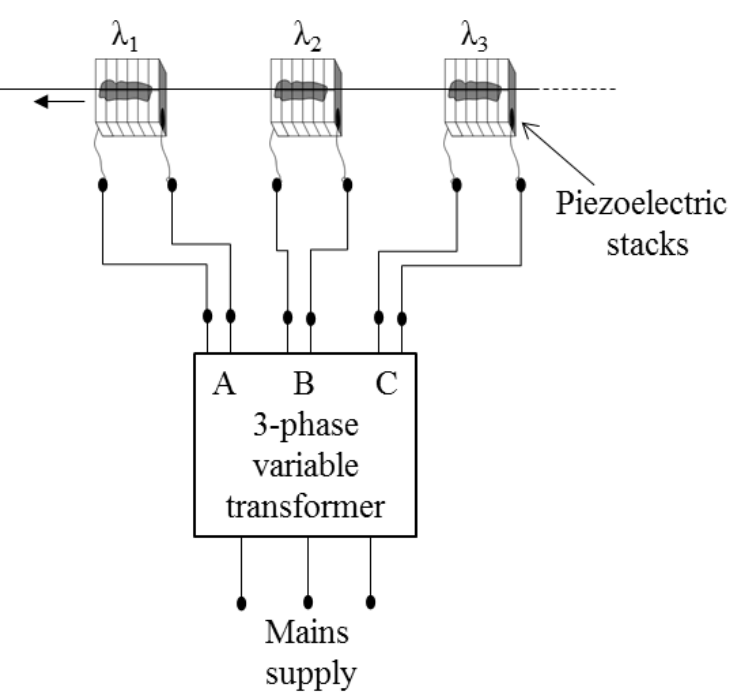

Fig. 4. Experimental construction of (a) prototype interrogation scheme and (b) test rig incorporating FBG sensors bonded to piezoelectric actuator stacks. CTR is 2-bit counter circuit. Three multiplexed FBGs are deployed (1535 nm, $1545 \mathrm{~nm}, 1555 \mathrm{~nm})$ corresponding to identical WDM channel centre wavelengths. 

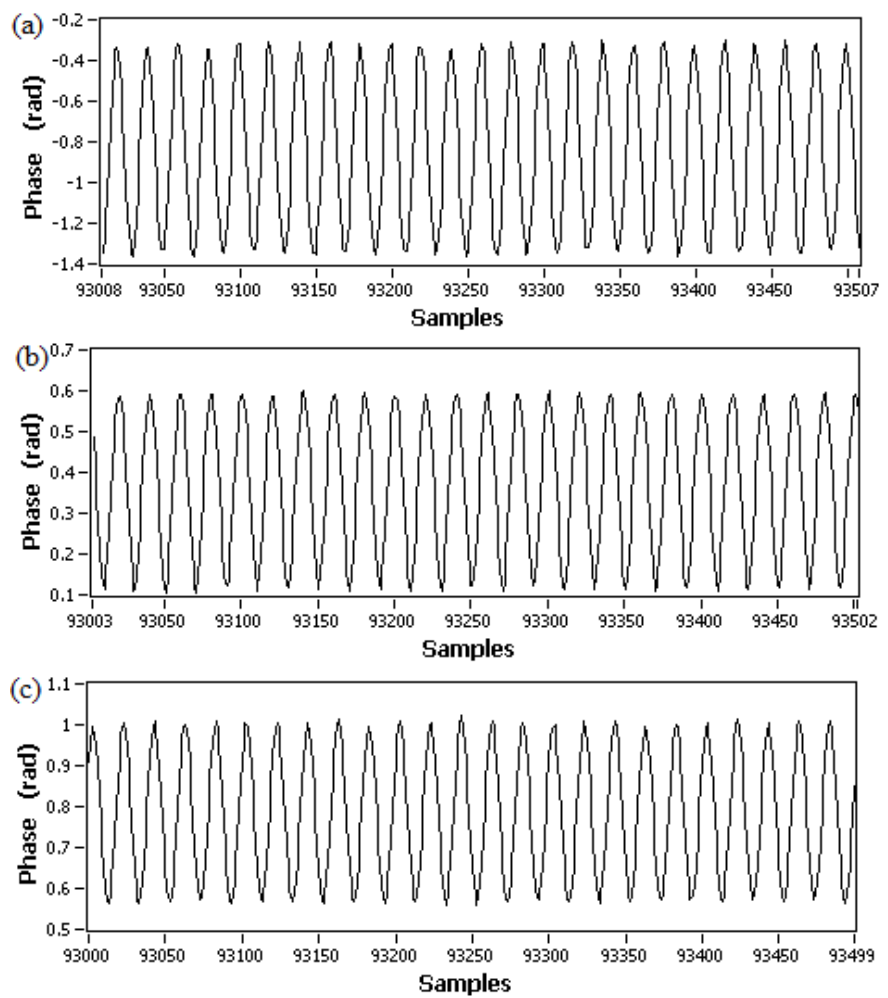

Fig. 5. Simultaneous interrogation of three FBG sensors ((a) $1535 \mathrm{~nm}$, (b) $1545 \mathrm{~nm}$, (c) $1555 \mathrm{~nm}$ ) configured to measure strain on piezoelectric stacks excited by 3-phase $50 \mathrm{~Hz}$ ac voltage. Strain on grating (a) is approximately 70 $\mu \varepsilon$ rms. Acquisition rate is $1 \mathrm{kHz}$ per sensor.

The noise spectral density of the interferometric technique was measured during a sensor quiescent period to be approximately $10 \mathrm{n} \varepsilon / \sqrt{\mathrm{Hz}}$, as can be seen approximately in Fig. 6. This contrasts with the microstrain resolution of higher end commercially available electronic schemes systems, and compares favourably with other interferometric FBG interrogators [17]. With improvements in acoustic noise isolation in the MZI unit it is likely that this resolution could yet be considerably improved. In addition, the use of sample averaging or filtering, based on the capability of this scheme to oversample channels, may be used to reduce noise levels in the scheme by an order of magnitude, although this is not demonstrated here.

To illustrate the lack of cross-talk while using this scheme, Fig. 7 (a) was recorded during application of a single strain signal at the $1535 \mathrm{~nm}$ sensor. It can be clearly seen from Fig. 7 (b), sampled simultaneously, that there is no trace of cross-talk between sensors on the spectra of the $1545 \mathrm{~nm}$ sensor.

\section{DISCUSSION}

Since the proposed architecture effectively trades the flexibility of scanned architectures for a rigid - and hence more quickly and easily controlled - selection mechanism for guidance of sensor reflections to the MZI, there exists a corresponding rigidity in sensor wavelength selection. However, in scanning WDM schemes [10], sensor wavelengths must also be pre-planned and should a sensor fail it must be replaced with one of identical wavelength. In practice therefore there is little reduction in flexibility using the present scheme, since in commercial installations the sensor numbers and locations are chosen at the design stage. The limitation in wavelength shift imposed by channel passbands is also mirrored in scanning schemes, where it is imposed instead by adjacent sensor wavelengths.

Since the incorporated passive MZI technique does not use feedback to set the operating point, signal components due solely to low-frequency drifts in the optical path difference may be monitored and subtracted from measurements to facilitate measurement of true dc signals. This can be achieved by the technique proposed in [10], or through incorporating a stable local wavelength in the multiplexed array.

It should be noted that although the laboratory demonstration achieved $4 \mathrm{KHz}$ multiplexing, using commercially-available switching technology multiplexing at up to $10 \mathrm{MHz}$ is feasible. The implication is that sensor multiplexing using this technique will be achievable at rates limited primarily by data processing capability, given that all other modules in the proposed scheme are passive.
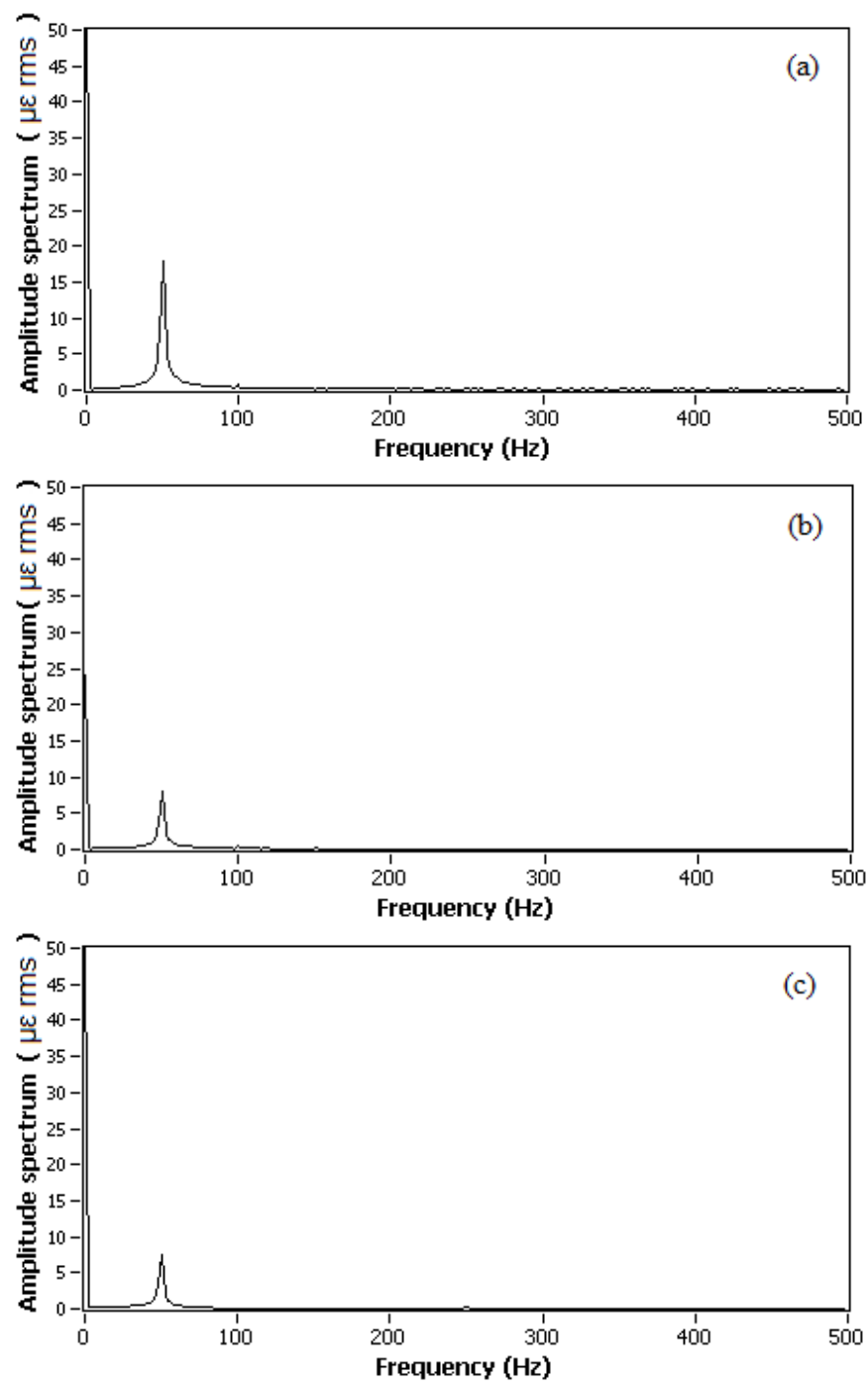

Fig. 6. Spectra of three sensor signals ((a) $1535 \mathrm{~nm}$, (b) $1545 \mathrm{~nm}$, (c) 1555 $\mathrm{nm})$ acquired at $1 \mathrm{kHz}$ each. Measuring strain induced on piezoelectric stacks by 3-phase ac $50 \mathrm{~Hz}$ voltage. Difference in signal amplitudes is due to variance in piezoelectric stack characteristics. 


\section{CONCLUSIONS}

In this article we have proposed a novel multiplexing interrogator for FBG sensors. The scheme incorporates passive modules for WDM and interferometric wavelength shift detection, and utilises fast optical path switching to enable large scale multiplexing. Through band-switching, the system is able to fully exploit the immediacy of the chosen passive interferometric technique, allowing for potentially extremely high speed sensor multiplexing using off-the-shelf components while retaining interferometric resolution.

A small-scale laboratory system was constructed in order to demonstrate $4-\mathrm{kHz}$ sensor multiplexing. No cross-talk was observed between sensor signals, with a noise floor of 10 $\mathrm{n} \varepsilon / \sqrt{ } \mathrm{Hz}$ achieved using a fusion-spliced interferometer with minimal noise isolation. The proposed system is solid state and simple to construct - incorporating minimal electronics and will be applicable to highly-demanding multi-point sensing applications where both measurement rate and resolution must be maximised.

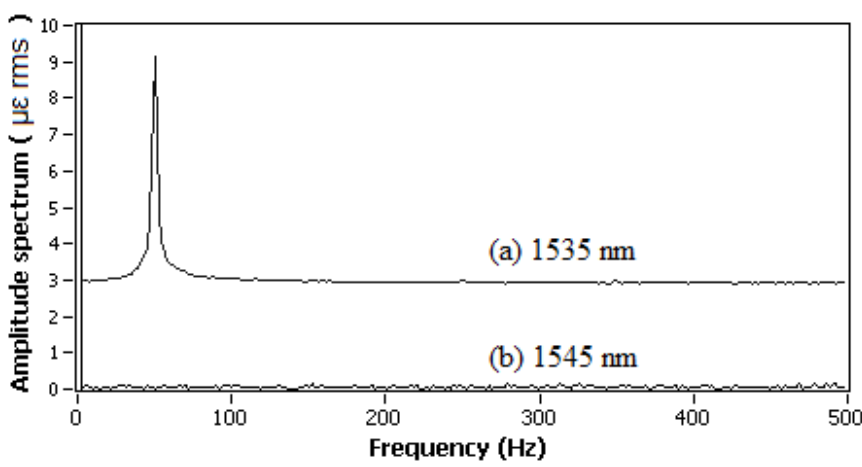

Fig. 7. Demonstration of robustness to cross-talk between multiplexed sensors during application of strain signal to one sensor (a) (offset on graph by $+3 \mu \varepsilon$ ). From (b) it can be seen that there is no trace of the applied signal.

\section{REFERENCES}

[1] G. Meltz, W. W. Morey, W. H. Glenn, "Formation of Bragg gratings in optical fibers by a transverse holographic method," Opt. Let., vol. 14, no. 15, pp. 823-825, August 1989.

[2] A. D. Kersey et al, "Fiber Grating Sensors," J. Lightwave Tech., vol. 15, no. 8, pp. 1442-1463, August 1997.

[3] P. Niewczas, J. R. McDonald, "Advanced optical sensors for power and energy systems," IEEE Instrumentation and Measurement Magazine, vol. 10, no. 1, pp. 18-28, February 2007.

[4] A. Mendez, "Fiber Bragg grating sensors: a market overview," Proc. SPIE, vol. 6619, p. 661905, 2007.

[5] A. Ezbiri, S. E. Kanellopoulos, and V. A. Handerek, "High resolution instrumentation system for fibre-Bragg grating aerospace sensors," Opt. Comm., vol. 150, pp. 43-48, May 1998.

[6] C. Boulet, D. J. Webb, M. Douay, and P. Niay, "Simultaneous interrogation of fiber Bragg grating sensors using an acoustooptic tunable filter," Phot. Tech. Lett., vol. 13, no. 10, pp. 1215-1217, November 2001.

[7] A. D. Kersey, T. A. Berkoff, and W. M. Morey, "Multiplexed fiber Bragg grating strain-sensor system with a fiber Fabry-Perot wavelength filter," Opt. Lett., vol. 18, pp. 1370-1372, 1993.

[8] A. D. Kersey, T. A. Berkoff, and W. M. Morey, "High resolution fiber Bragg grating based strain sensor with interferometric wavelength detection," Elec. Lett., vol. 28, no. 236, p. 237, 1992.

[9] T. A. Berkoff and A. D. Kersey, "Fiber Bragg grating array sensor system using a bandpass wavelength division multiplexer and interferometric detection," Phot. Tech. Lett., vol. 8, no. 11, pp. 15221524, 1996.

[10] G. A. Johnson, M. D. Todd, B. L. Aulthouse and C. C. Chang, "Fiber Bragg grating interrogation and multiplexing with a $3 \times 3$ coupler and a scanning filter," J. Lightwave Tech., vol. 18, no. 8, pp. 1101-1105, August 2000.

[11] M. D. Todd, G. A. Johnson and C. C. Chang, "Passive, light intensityindependent interferometric method for fibre Bragg grating interrogation," Elec. Lett., vol. 35, no. 22, pp. 1970-1971, October 1999.

[12] M. D. Todd, M. Seaver, and F. Bucholtz, "Improved, operationallypassive interferometric demodulation method using $3 \times 3$ coupler," Elec. Lett., vol. 38, no. 15, pp. 784-786, 2002.

[13] F. Schleip, R. Hewith, and G. Schiffner, "Phase sensitive investigations of 3x3 singlemode fibre directional couplers", Elec. Lett., vol. 29, pp. $68-70,1993$

[14] EPI Photonics, www.epiphotonics.com, [Aug. 18, 2011].

[15] Y. J. Rao, "In-fibre Bragg grating sensors," Meas. Sci. Tech., vol. 8, pp. 355-375, 1997.

[16] P. Orr and P. Niewczas, "An optical fibre system design enabling simultaneous point measurement of magnetic field strength and temperature using low-birefringence FBGs," Sensors and Actuators: A., vol. 163, pp. 68-74, 2010.

[17] B. Lee, "Review of the present status of optical fiber sensors," Optical Fiber Technology, vol. 9, pp. 57-79, 2003.

Philip Orr (S'07) received the BEng $\left(1^{\text {st }}\right.$ Class Hons.) degree in electronic and electrical engineering from the University of Strathclyde, Glasgow, U.K., in 2007 where he is presently working towards the $\mathrm{PhD}$ degree in the area of optical fibre sensors.

His research interests include fibre sensor interrogation schemes, electromagnetic field measurement, nuclear diagnostics, and power system protection.

Pawel Niewczas (M'05) received the MSc degree in Electrical Engineering from Technical University of Lublin in 1995 and the $\mathrm{PhD}$ degree in the area of optical current sensors from the University of Strathclyde, Glasgow in 2000.

$\mathrm{He}$ is currently a Senior Lecturer in the Department of Electronic and Electrical Engineering, University of Strathclyde, and is leading the Advanced Sensors Team within the Institute for Energy and Environment in the same department. His main interests centre on the advancement of optical sensing methods in such areas as power system metering and protection; gas turbine monitoring; downhole pressure, temperature, voltage and current measurement; sensing in nuclear fission and fusion environments; and other sensing applications. He has published over 60 technical papers in this area. 